\title{
Efficient gene editing in Corynebacterium glutamicum using the CRISPR/Cas9 system
}

Feng Peng ${ }^{1,2,3}$, Xinyue Wang ${ }^{1,2,3}$, Yang Sun ${ }^{1,2,3}$, Guibin Dong ${ }^{1,2,3}$, Yankun Yang ${ }^{1,2,3}$, Xiuxia Liu ${ }^{1,2,3^{*}}$ and Zhonghu Bai ${ }^{1,2,3^{*}}$

\begin{abstract}
Background: Corynebacterium glutamicum (C. glutamicum) has traditionally been used as a microbial cell factory for the industrial production of many amino acids and other industrially important commodities. C. glutamicum has recently been established as a host for recombinant protein expression; however, some intrinsic disadvantages could be improved by genetic modification. Gene editing techniques, such as deletion, insertion, or replacement, are important tools for modifying chromosomes.
\end{abstract}

Results: In this research, we report a CRISPR/Cas9 system in C. glutamicum for rapid and efficient genome editing, including gene deletion and insertion. The system consists of two plasmids: one containing a target-specific guide RNA and a homologous sequence to a target gene, the other expressing Cas9 protein. With high efficiency (up to 100\%), this system was used to disrupt the porB, mepA, clpX and Ncgl0911 genes, which affect the ability to express proteins. The porB- and mepA-deletion strains had enhanced expression of green fluorescent protein, compared with the wild-type stain. This system can also be used to engineer point mutations and gene insertions.

Conclusions: In this study, we adapted the CRISPR/Cas9 system from S. pyogens to gene deletion, point mutations and insertion in C. glutamicum. Compared with published genome modification methods, methods based on the CRISPR/Cas9 system can rapidly and efficiently achieve genome editing. Our research provides a powerful tool for facilitating the study of gene function, metabolic pathways, and enhanced productivity in C. glutamicum.

Keywords: CRISPR/Cas9, Corynebacterium glutamicum, Genome editing, Protein expression

\section{Background}

Corynebacterium glutamicum, a gram-positive bacterium with a high $\mathrm{G}+\mathrm{C}$ content, has been used for the industrial production of various amino acids for more than 50 years. Moreover, it has recently demonstrated strong potential for use as a protein expression system [1, 2] because of its excellent culture characteristics and also because it is non-pathogenic, does not produce endotoxins, and is generally recognized as safe [3, 4]. Furthermore, $C$. glutamicum produces minimal protease activity

\footnotetext{
*Correspondence: liuxiuxia@jiangnan.edu.cn;

baizhonghu@jiangnan.edu.cn

${ }^{1}$ National Engineering Laboratory for Cereal Fermentation Technology,

Jiangnan University, Wuxi 214122, China

Full list of author information is available at the end of the article
}

in the culture supernatant and has the ability to secrete properly folded proteins, which can improve subsequent purification efficiency. However, compared with Escherichia coli (E. coli), C. glutamicum has some intrinsic disadvantages, e.g. much lower transformation efficiency and lower levels of protein expression $[4,5]$; comprehensive genetic and physiological investigations are needed so that C. glutamicum can fulfill its potential $[6,7]$. To achieve this, rapid and efficient genome editing methods suitable for C. glutamicum are needed.

Integrative plasmid vectors have been developed for gene deletion, mutation and insertion. These include suicide plasmids based on $\mathrm{SacB}$, which hydrolyzes sucrose and synthesizes levan, leading to sucrose sensitivity in C. glutamicum $[8,9]$, and suicide plasmids based on the 
Cre/loxP system, in which Cre recombinase catalyzes specific recombination between two loxP sites $[10,11]$. However, the efficiency of this gene deletion method is not very efficient because two rounds of homologous recombination are required and mutant selection after the second recombination is time-consuming $[12,13]$. Therefore, a more efficient method for C. glutamicum genome editing is desirable.

The recent development of the CRISPR/Cas9 system provides a simple, sequence-specific platform for genome engineering $[14,15]$. The widely used Streptococcus pyogenes (S. pyogenes) type II CRISPR/Cas9 system, which requires a mature CRISPR RNA (crRNA), a trans-activating CRISPR RNA (tracrRNA), and a DNA endonuclease, Cas9, has been harnessed for targeted genome editing in many organisms [15-17]. The Cas9 protein is an RNA-guided endonuclease that cleaves target DNA; a 20 bp complementary region (N20) within the crRNA can guide Cas9 to its specific target $[18,19]$. The $20 \mathrm{nt}$ sequence, known as the protospacer [20], contains a specific protospacer-adjacent motif (PAM) at its $3^{\prime}$ end [21]. The PAM sequence leads Cas9 to create a double-strand break (DSB) at the target sequence, and the DSB stimulates the DNA repair pathway by non-homologous end joining (NHEJ) or homolog-directed repair (HDR) [2224]. The crRNA and tracrRNA can be fused together to generate a single synthetic guide RNA (sgRNA), which simplifies genome editing design [25].

The CRISPR/Cas9 system, has been widely applied in both prokaryotes and eukaryotes, such as E. coli [26-28], Saccharomyces cerevisiae [29], Staphylococcus aureus [25], Bacillus subtilis [30], Lactococcal Phages [17], higher-plants [31, 32], and animal cells [21, 33]. Moreover, the system has been used in C. glutamicum to manipulate the expression levels of specific genes [12], but not for genome editing, such as gene deletion or insertion, or the generation of point mutations. A C. glutamicum genome editing tool based on the CRISPR-Cpf1 system was recently reported; however, the authors of this study were unsuccessful in developing a CRISPR/Cas9-based system for use in C. glutamicum [34]. Here, we developed a CRISPR/Cas9-based genome editing method to investigate the function of C. glutamicum genes involved in recombinant protein expression. We analyzed the transcriptomes of $C$. glutamicum grown under different dissolved oxygen conditions to identify genes that might affect substance and energy metabolism and, therefore, might play important roles in the ability of C. glutamicum to express recombinant proteins [35]. We identified four genes, porB, mepA, $\operatorname{clpX}$, and Ncgl0911, and used the system to disrupt them in C. glutamicum ATCC 13032 to investigate their endogenous functions and their effects on recombinant protein production. Highly efficient gene deletions were obtained via homolog-directed repair through the introduction of a DNA repair template. In addition, point mutations and gene insertions were achieved with an efficiency of 100 and $66.7 \%$, respectively. We also expanded the system to C. glutamicum CGMCC1.15647, a host strain for recombinant protein production. Green fluorescent protein (GFP) was used as a model protein to examine the effect of different genedeletion strains produced by the CRISPR/Cas9 system on recombinant protein expression and the results showed varying GFP expression levels in different strains. Overall, our CRISPR/Cas9-based genome editing method enabled rapid and efficient genome editing in a single step without the need for a marker gene, making this an effective tool for gene analysis and genome engineering in $C$. glutamicum.

\section{Results}

Construction of the CRISPR/Cas9 system in two plasmids

To establish a genome editing system in C. glutamicum based on CRISPR/Cas9, we designed and constructed a two-plasmid system that separated Cas9 and the sgRNA into pFSC and pFST plasmid series, respectively (Fig. 1a and b). pFSC was constructed from pXMJ19 [36], and included the Cas9 gene, an SD sequence and the Ptac promoter. We chose the strong Ptac promoter to drive the expression of Cas9, which is IPTG-inducible. The SD sequence (AAAGGAGGACAACTA) in front of the Cas9 gene ATG is indispensable for Cas9 protein expression. pFST was constructed in the temperature sensitive pECXK99E backbone [37] containing the temperature sensitive repA from pDTW109 [9], which enables fast curing of the plasmid after editing. An sgRNA containing an N20 sequence targeting the genomic locus of interest under control of the IPTG-inducible Ptrc promoter and a homologous repair template of the target gene were inserted into pEC-XK99E to give pFST. The homologous repair template regions upstream and downstream of the target locus were generated by PCR and assembled into pFST using an NEB Gibson assembly cloning kit. sgRNA was ligated into EcoRI and $X b a \mathrm{I}$ cloning sites of pFST, while the repair template was inserted into the BglII site. pFSC and pFST carry kanamycin and chloramphenicol resistance genes, respectively.

\section{Genome editing in C. glutamicum ATCC 13032 and C. glutamicum CGMCC1.15647}

To test the CRISPR/Cas9 system, the porB gene was chosen to be knocked out. This gene is an anionselective channel that affects substance exchange in $C$. glutamicum [11, 38]. The sgRNA containing a 20 bp spacer was designed from the por $B$ gene sequence and was checked to have no predicted off-target effects by 

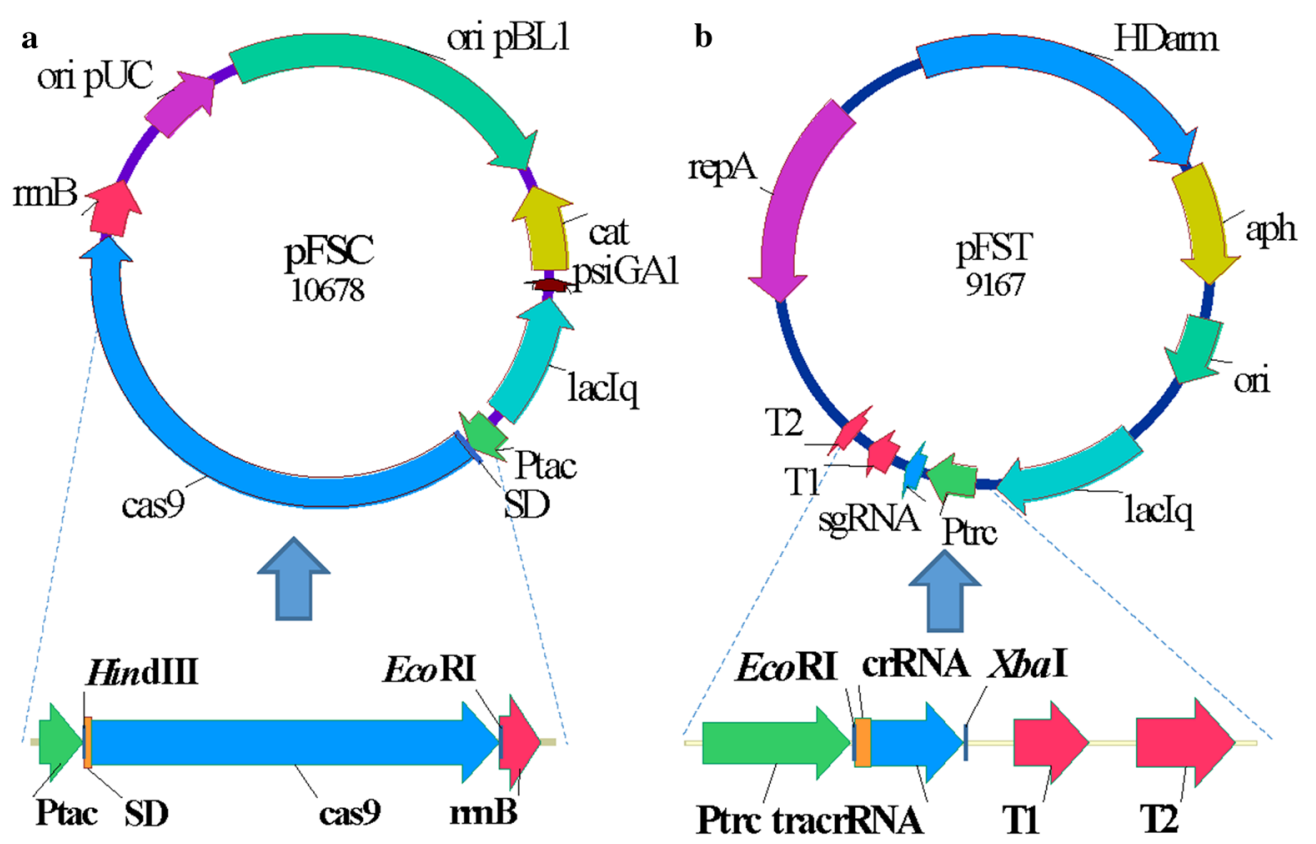

c EcoRI

5'-GAATTC(N20)GTTTTAGAGCTAGAAATAGCAAGTTAAAA TAAGGCTAGTCCGTTATCAACTTGAAAAAGTGGCACCGAG TCGGTGCTTTTTTGGCTGTTTTGGCGGATGAGAGAAGATTT TCAGCCTGATACAGATTAAATTCTAGA -3'

\section{$X b a \mathrm{I}$}

Fig. 1 Design of the CRISPR/CAS9 system for gene deletion in C. glutamicum. a Strategy for construction of pFSC. Cas9 is controlled by the IPTGinducible Ptac promoter, the SD sequence is designed to enhance the expression of Cas9; $\mathbf{b}$ strategy for construction of pFST. The sgRNA cassette is under the control of the IPTG-inducible Ptrc promoter, the $20 \mathrm{nt}$ target sequence is shown in gold, the backbone is a temperature sensitive repA replicon, the HDarm is ligated into pFST at the Bgll site; c strategy for construction of the sgRNA. The red N20 is the 20 nt target sequence, and the underlined sequences are the sgRNA scaffold. The EcoRI and Xbal sites are used to assemble the sgRNA into pFST

BLAST searches against the C. glutamicum genomic sequence (https://blast.ncbi.nlm.nih.gov/Blast.cgi). The sgRNA sequence and the gene length are shown in Table 1. The sgRNA was subcloned into pFST, and after sequence validation, a correct plasmid named pFST-porBsgRNA was selected for transformation. First we transformed pFSC and pFST-porBsgRNA into C. glutamicum ATCC 13032. The sgRNA directed the Cas9 endonuclease to the porB locus, and the sgRNA/ Cas9 complex generated a DSB at the locus. However, no colonies with the plasmid containing the sgRNA were observed after transformation, whereas more than $1 \times 10^{3}$ colonies were obtained with the plasmid without the sgRNA (Additional file 1: Figure S1), indicating that the system containing only sgRNA and Cas9 was not effective in C. glutamicum. The DSB can be repaired by error prone NHEJ, but the efficiency of NHEJ in $C$. glutamicum is much lower than that in eukaryotic organisms. We, therefore, assembled the repair arms into pFST-porBsgRNA to assess editing efficiency by fixing the DSB through homolog-directed repair. In addition, three negative controls were used: the first was pFST-porB containing an sgRNA targeting the por $B$ sequence and a homologous repair template, the second was pFST-porBT without an sgRNA, and the third was pFST-porBsgRNA without a homologous repair template. After transformation of the three plasmids, only the first produced DNA knock out. The plasmid not containing an sgRNA was wild-type, and no clones were obtained from the third plasmid because of the absence of the homology-directed repair template. As shown in Table 2 and Fig. 2b, the efficiency of deleting porB 
Table 1 The PAM site and the sgRNA sequence used in this study

\begin{tabular}{|c|c|c|c|c|}
\hline Gene & PAM sequence & PAM site and gene length & Target strand & GC (\%) \\
\hline porB & GGAGGATAGGTTTGCGAAGTCGG & $83 / 381$ & NT & 50 \\
\hline mepA & GGCACCTTCACCTCAGGATTCGG & $214 / 576$ & $\mathrm{~T}$ & 55 \\
\hline$c l p X$ & GGCTGAAATCTCCGACGGCTIGG & $194 / 1281$ & NT & 60 \\
\hline Ncgl0911 & GGTAACTGGGCTGGCCAAAAGGG & $62 / 1260$ & NT & 55 \\
\hline mepA1 & GGTGGCGGTAGCGGTTGCGGTGG & $62 / 576$ & NT & 75 \\
\hline mepA2 & GGATGCTGGTGCCATGGTGGCGG & $77 / 576$ & NT & 65 \\
\hline mерАЗ & GGAGGAAAGGCCTGCGTAGTCGG & $108 / 576$ & NT & 60 \\
\hline mepA4 & GGCGGCTGGTGCGACGGCGGTGG & $173 / 576$ & NT & 85 \\
\hline mepA5 & GGCCAGGAAATCGCAGGAATGGG & $445 / 576$ & $\mathrm{~T}$ & 55 \\
\hline mерА6 & GGCAGCCAAGGATTCTCCACCGG & $467 / 576$ & $\mathrm{~T}$ & 60 \\
\hline
\end{tabular}

The PAM sites are shown in underline. NT and T mean sgRNAs targeting to the template (T) or nontemplate (NT) respectively

Table 2 Results of the porB deletion in C. glutamicum ATCC 13032 and C. glutamicum CGMCC1.15647

\begin{tabular}{llllll}
\hline No & Host cell & Plasmid & Element & Results (D/W/T) & Efficiency (\%) \\
\hline 1 & ATCC 13032 & pFSC & Cas9 & $0 / 5 / 5$ & 0 \\
2 & ATCC 13032 & pFST-porBsgRNA & sgRNA & $0 / 5 / 5$ & 0 \\
3 & ATCC 13032 & pFST-porBT & Hdarm & $0 / 5 / 5$ & 0 \\
4 & ATCC 13032 & pFSC, pFST-porBT & Cas9 + Hdarm & $0 / 5 / 5$ & 0 \\
5 & ATCC 13032 & pFSC, pFST-porBsgRNA & Cas9 + sgRNA & $0 / 0 / 0$ & 0 \\
6 & ATCC 13032 & pFST-porB & sgRNA + Hdarm & $0 / 6 / 6$ & 0 \\
7 & ATCC 13032 & pFSC, pFST-porB & Cas9 + sgRNA + Hdarm (1000 bp) & $18 / 0 / 18$ & 100 \\
8 & CGMCC 1.15647 & pFSC, pFST-porB15647 & Cas9 + sgRNA + Hdarm (1000 bp) & $16 / 0 / 16$ & 100 \\
9 & ATCC 13032 & pFSC, pFST-porB600 & Cas9 + sgRNA + Hdarm (600 bp) & $10 / 2 / 12$ & 83.3 \\
10 & ATCC 13032 & pFSC, pFST-porB300 & Cas9 + sgRNA + Hdarm (300 bp) & $10 / 2 / 12$ & 83.3 \\
11 & ATCC 13032 & pFSC, pFST-porB100 & Cas9 + sgRNA + Hdarm (100 bp) & $2 / 10 / 12$ & 16.7
\end{tabular}

$\mathrm{D}$, number of colonies that harbored gene-deleted cells; W, number of colonies that harbored wild type cells; T, total number of colonies used for PCR screening; Efficiency, probability of deletion events occurring, calculated as D/T * 100\%; ATCC 13032, C. glutamicum ATCC 13032; CGMCC 1.15647, C. glutamicum CGMCC1.15647

by this system was $100 \%$, confirmed by both the PCR screening and sequencing.

To further assess the potential use of this system, we applied it to C. glutamicum CGMCC1.15647, a host strain for recombinant protein expression. As in C. glutamicum ATCC 13032, the efficiency was 100\% (Fig. 2c). These observations indicated that the CRISPR/Cas9 system indeed deleted the porB gene in C. glutamicum.

\section{Different repair template fragments}

To evaluate the effect of repair arm size on gene editing because the length of homologous arms affects recombination frequency. We constructed a series of donor templates with homologous arms of varied length (0.6, 0.3 and $0.1 \mathrm{~kb}$ ) which could be easily synthesized and assembled. The vectors were constructed by assembling the repair arms into pFST-porBsgRNA and were then transformed into C. glutamicum ATCC 13032. Then we used PCR and sequence analysis to validate the deletions. As shown in Table 2 and Fig. 3, efficiency with the 0.3 and
$0.6 \mathrm{~kb}$ arms was $83.3 \%$, which is lower than the efficiency with $1 \mathrm{~kb}$ arms. However, the efficiency with $0.1 \mathrm{~kb}$ arms was only $16.7 \%$.

\section{Editing different genes}

To further validate the system, three other C. glutamicum genes were targeted. These were mepA, $\operatorname{clpX}$ and Ncgl0911, which encode genes involved in cell wall metabolism, proteolysis and the two-component system, respectively [39-42]. We constructed the knockout plasmids, pFST-mepA, pFST-clpX and pFST-0911, and used them to disrupt mepA, $\operatorname{clpX}$ and Ncgl0911 genes using the above CRISPR/Cas9 method. The proportion of mutants in the transformants was determined by PCR and sequencing. The PAM site and the gene length are shown in Table 1 . We showed above that 300 bp repair arms are sufficient for gene deletion; therefore, we amplified $300 \mathrm{bp}$ left and right arms separately by PCR and assembled them into pFST (Fig. 4a). For mepA, two pure mutant and two mixed mutant and wild-type populations 


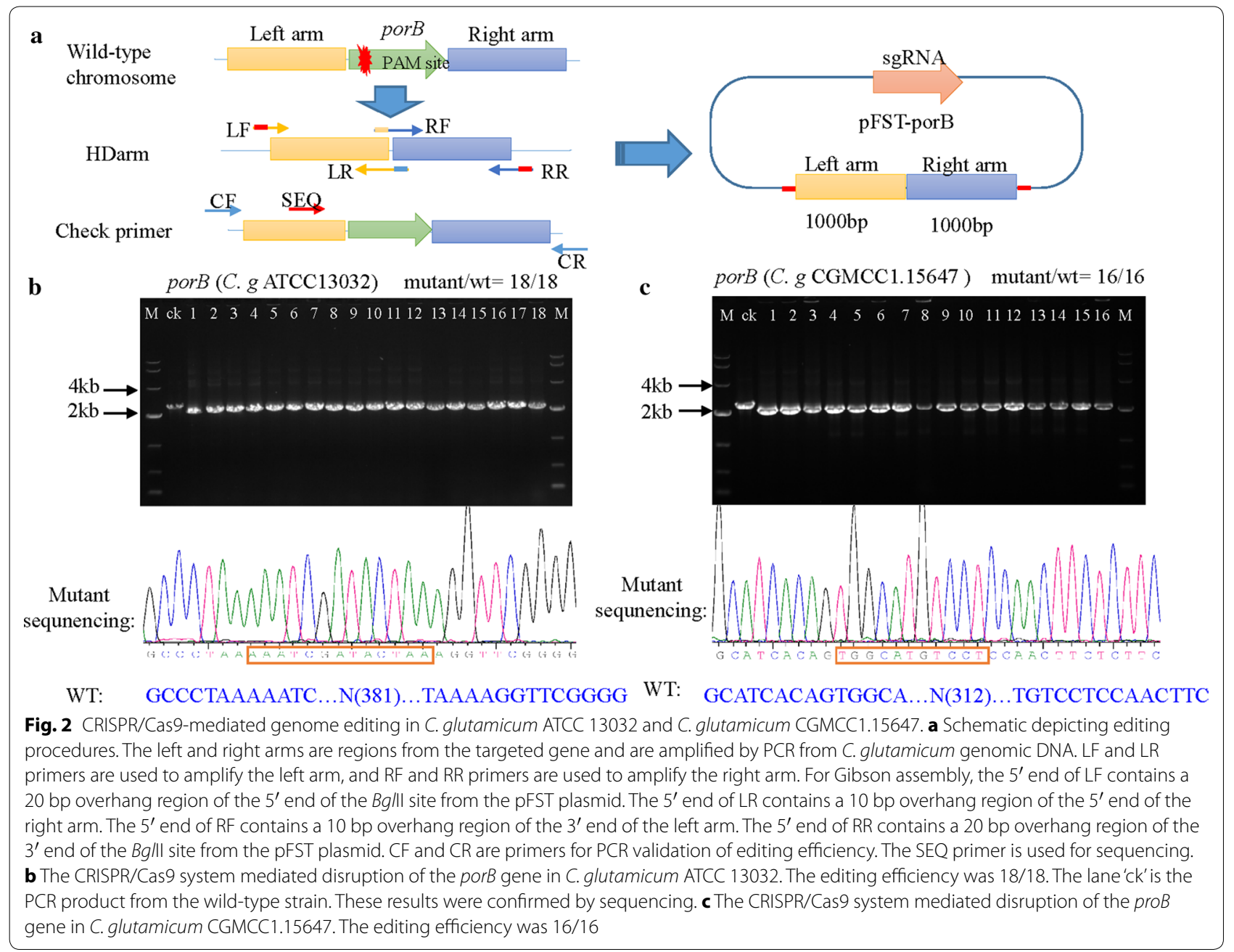

were observed from 15 colonies (Fig. 4b). In contrast, no mixed colonies were detected for $\operatorname{clp} X$ and Ncgl0911 and the mutation efficiency was $5 / 16$ and $4 / 15$, respectively (Fig. 4c and d). These results demonstrated the high efficiency of this system for gene deletion in C. glutamicum ATCC 13032.

\section{Point mutation and gene insertion in C. glutamicum}

The study of gene functions can often be facilitated by the generation of point mutations in a target gene. We, therefore, designed this system to mutate specific sites in the genome. We first created a six base mutagenic site in the repair template and assembled it into pFST-m (Fig. 5a). Next, we transformed the plasmid into C. glutamicum ATCC 13032. We then used PCR and sequencing to validate the mutation. A high editing efficiency of $6 / 6$ was observed (Fig. 5c).

Analysis of gene functions and pathways may also need the insertion of gene(s) into the genome, such as a reporter system to monitor the activity of a promoter of interest. To test this system for creating insertion mutants, we assembled the GFP gene $(g f p)$ and repair arms into pFST-i (Fig. 5b). The repair arm length was $1000 \mathrm{bp}$. After transforming the plasmid into C. glutamicum ATCC 13032, we observed a high editing efficiency of $8 / 12$ (Fig. 5d). The efficiency of gene insertion for $300 \mathrm{bp}$ repair arms was not as high as that for $1000 \mathrm{bp}$ arms at only $3 / 12$, possibly owning to the insert gene being longer than the repair arms (Additional file 1: Figure S2).

\section{Effect of different sgRNAs on editing efficiencies}

The efficiency of editing the mepA gene was not as high as that for other genes, showing that gene editing efficiencies may be affected by different sgRNAs targeting different sites and different strands of the same gene. To improve efficiency, we tested different sgRNAs targeting different sites and different strands of the mepA gene. The PAM site and the sgRNA sequence are shown in Table 1. After transformation of the plasmid into C. glutamicum 


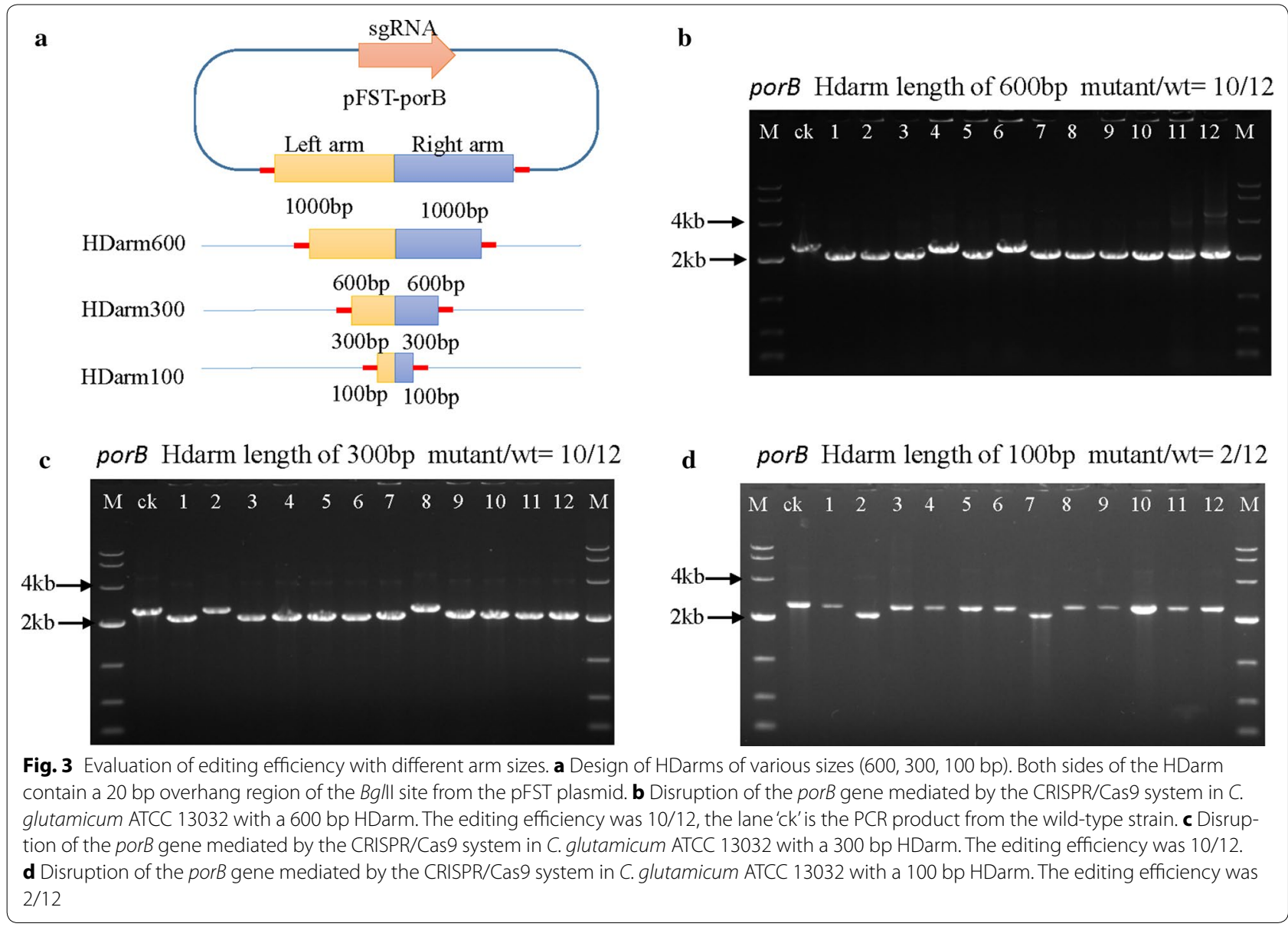

ATCC 13032, we used PCR and sequencing to validate the deletion. As shown in Table 3 and Additional file 1: Figure S3, the efficiencies of sgRNAs $1-6$ were $3 / 12$, 0/12, 10/12, 4/12, 12/12 and 6/12, respectively. Repair arms of the same length but binding to different sgRNAs were used and generated different editing efficiencies, increased the efficiencies from 13.3 to $100 \%$. These results indicated that different sgRNAs have a great influence on editing efficiency and that it is better to use an sgRNA with the GC content under $60 \%$.

\section{Off target analysis of edited strains}

To analysis the off target effect in C. glutamicum after gene editing by CRISPR/Cas9 system, genome resequencing were performed to identify all the single nucleotide polymorphism (SNP) and insertions and deletions (Indel) using the proB-deleted strain and the mepAdeleted strain with wild type C. glutamicum ATCC13032 as the negative control. In addition, to analysis whether the Cas9 protein create the off target without the sgRNA, the SNP and Indel of the wild type strain containing Cas9 protein were also detected. Result showed no off-target mutations were detected in the wild type strain containing Cas9 protein, that is, compared with the wild type the SNP and Indel were not detected in this strain. Meanwhile, in the mepA-deleted strain no SNP and Indel were detected, while in the porB-deleted strain no SNP but 1 Indel with 1 base deleted was identified (Additional file 1: Table S3). The results suggested the CRISPR/Cas9 gene editing system with HDR can generate the off target in a very low probability.

\section{Quantification of GFP activity}

The four C. glutamicum genes, porB, mepA, $\operatorname{clp} X$ and Ncgl0911, encoding genes involved in cell wall metabolism, anion absorption, proteolysis, and the two-component system, were identified as differentially expressed under different dissolved oxygen levels by the analysis of transcriptome data (Accession Number GSE77502). Dissolved oxygen is an important factor that significantly influences metabolism and recombinant protein product yield in C. glutamicum when cultured in a bioreactor [35]. We, therefore, speculated that these genes play important roles in affecting recombinant protein 


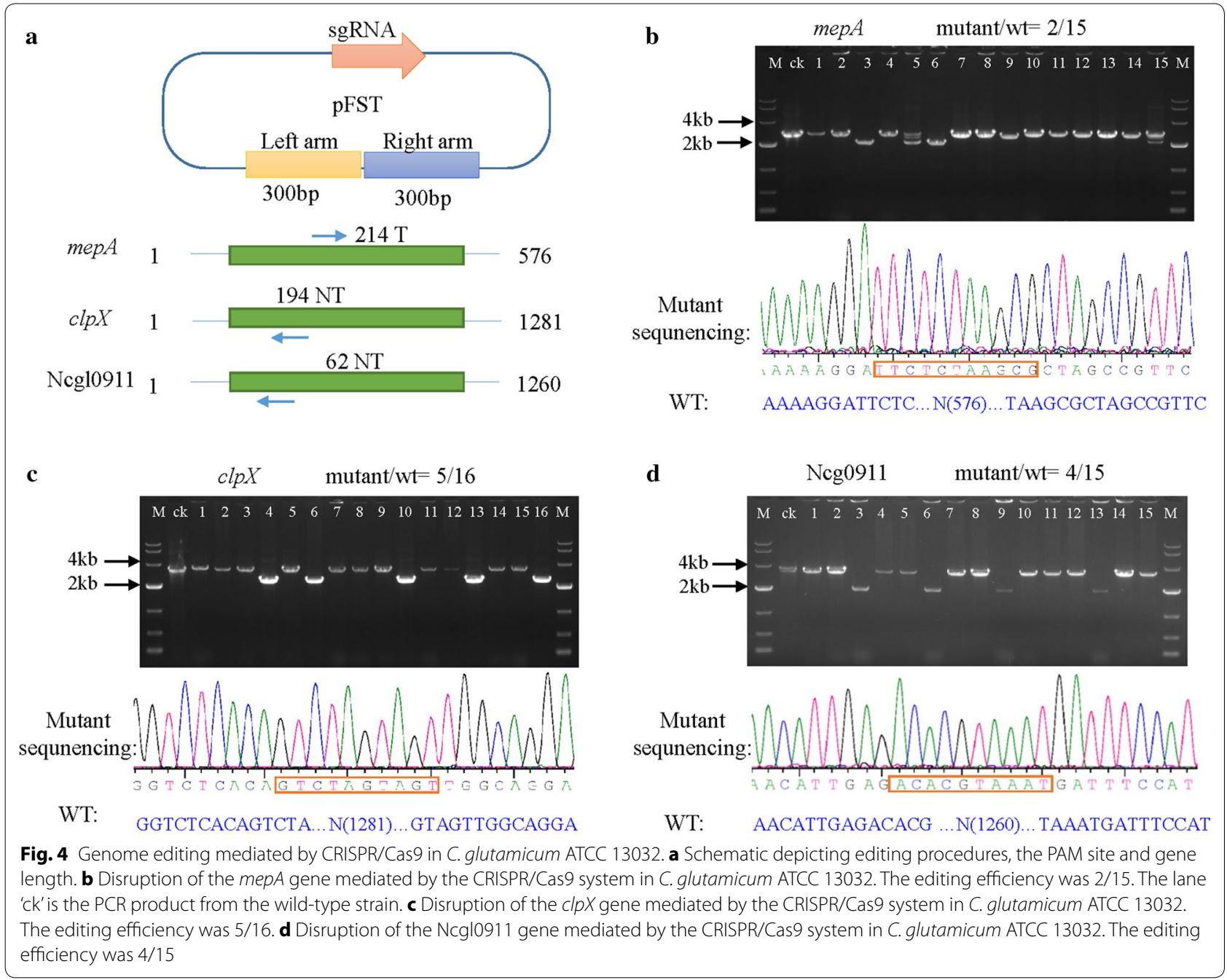

expression in C. glutamicum. In our study, GFP was chosen as a model protein to evaluate the gene deletion strains. We removed the lacIq gene from pXMJ19 to create a constitutive expression vector. We then constructed the GFP expression plasmid, pXMJ19-EGFP, by introducing the $g f p$ gene into pXMJ19 (Additional file 1: Figure S4). The phenotype of the generated mutants was confirmed by genetic complementation, here, we used the plasmid pECXK99 as a borne to complement the deleted gene (Additional file 1: Figure S5). After transforming the plasmid into C. glutamicum ATCC 13032, the gene deletion strains and the complementation strains, we cultured the different strains and measured their $\mathrm{OD}_{600}$ values and fluorescence intensities. The results indicated impaired growth of the $c l p X$ mutant compared with the wild-type strain, while the other mutant strains showed the same growth rate as the wild type (Fig. 6). The complementation strains of porB, mepA and Ncgl0911 showed the same growth rate as the wild type and mutant strains (Additional file 1: Figure S6). The $c l p x$ gene deletion strain can't survival in the medium for competent cell, so we can't get the complementation strain of clpx gene. The mep $A$ and por $B$ mutants showed 55.2 and $62.4 \%$ greater GFP fluorescence respectively, compared with wild type (Fig. 7, Additional file 1: Figure S7), but the reason for this requires further study. This CRISPR/ Cas 9 system provides an efficient way to study the function of different genes and identified a key gene involved in recombinant protein expression.

\section{Discussion}

In this study, we adapted the CRISPR/Cas9 system from $S$. pyogens to be efficient at generating gene deletions, point mutations and gene insertions in C. glutamicum ATCC 13032 and C. glutamicum CGMCC1.15647. Compared with published genome modification methods, methods based on the CRISPR/Cas9 system can fast and accurate achieve genome editing. 


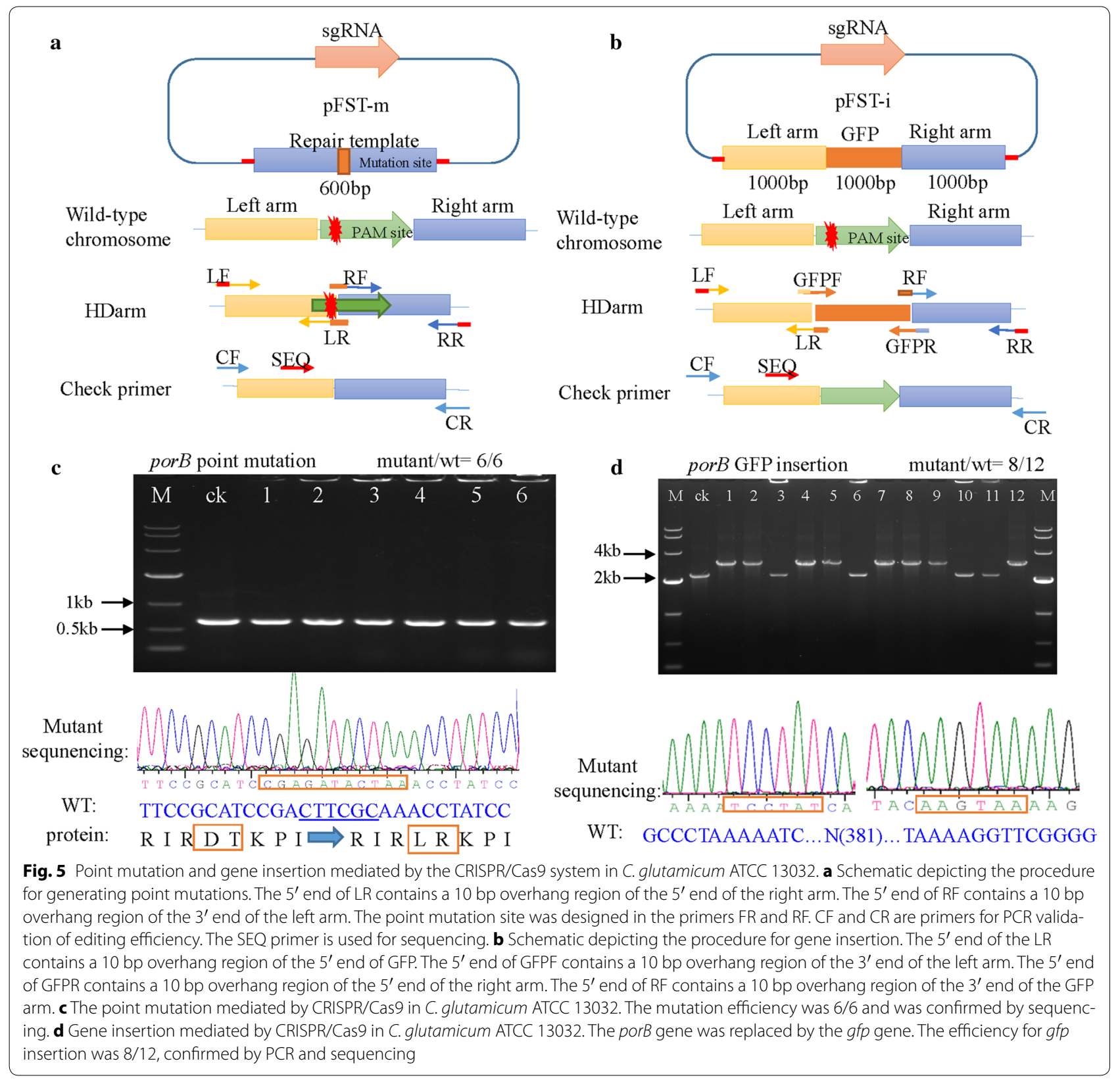

Table 3 Results of the mepA deletion in C. glutamicum ATCC 13032

\begin{tabular}{llll}
\hline Plasmid & Element & Results (D/W/T) & Efficiency (\%) \\
\hline pFSC, pFST-mepA1 & Cas9 + sgRNA1 + Hdarm (300 bp) & $3 / 9 / 12$ & 25 \\
pFSC, pFST-mepA2 & Cas9 + sgRNA2 + Hdarm (300 bp) & $0 / 12 / 12$ & 0 \\
pFSC, pFST-mepA3 & Cas9 + sgRNA3 + Hdarm (300 bp) & $10 / 2 / 12$ & 83.3 \\
pFSC, pFST-mepA4 & Cas9 + sgRNA4 + Hdarm (300 bp) & $4 / 8 / 12$ & 33.3 \\
pFSC, pFST-mepA5 & Cas9 + sgRNA5 + Hdarm (300 bp) & $12 / 0 / 12$ & 100 \\
pFSC, pFST-mepA6 & Cas9 + sgRNA6 + Hdarm (300 bp) & 50 \\
\hline
\end{tabular}

$\mathrm{D}$, number of colonies that harbored gene-deleted cells; $\mathrm{W}$, number of colonies that harbored wild type cells; $\mathrm{T}$, total number of colonies used for PCR screening; Efficiency, probability of deletion events occurring, calculated as D/T * $100 \%$ 


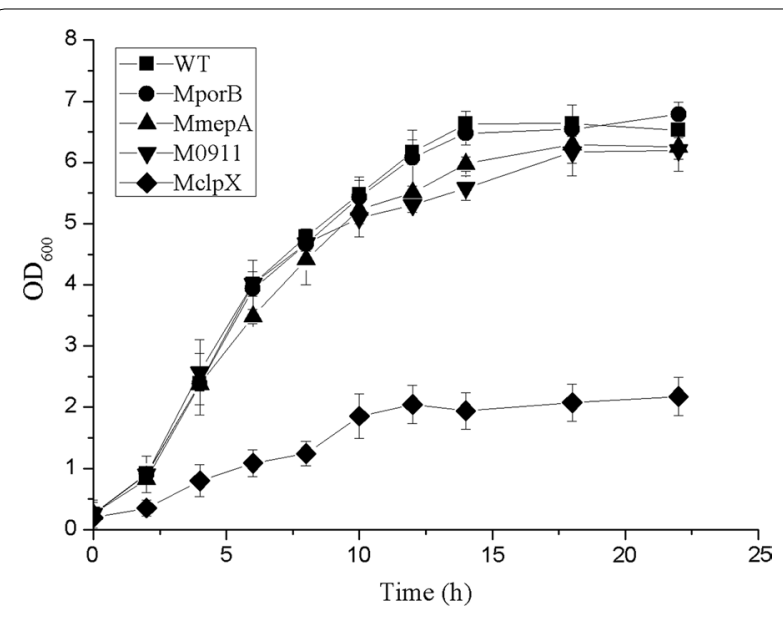

Fig. 6 Growth phenotypes of the porB, mepA, clpX and Ncgl0911deleted strains. WT, wild-type strain; MporB, porB-deleted mutant; MmepA, mepA-deleted mutant; M0911, Ncgl091 1-deleted mutant; MclpX, clpX-deleted mutant; the clpX mutant showed impaired growth compared with the wild-type strain while the other mutants showed the same growth rate as the wild-type. Date are representative of triplicate cultures

In this study we observed that C. glutamicum cannot survive after the DNA DSBS introduced by Cas9 in the absence of a homologous recombination template. Because the efficiency of NHEJ in C. glutamicum is much lower than that in eukaryotic organisms (Additional file 1: Figure S1). So a homologous recombination template is necessary in this system [13]. We observed nearly $100 \%$ efficiency for porb gene deletion in C. glutamicum ATCC 13032 and C. glutamicum CGMCC1.15647 when a homology-based repair template was supplied. The system is readily applicable to other related Corynebacteria.

Typically, relatively long flanking regions of at least $700 \mathrm{bp}$ are used for incorporation of novel genetic elements into the C. glutamicum genome [27]. A powerful genome editing tool should have a high efficiency and be independent of a marker gene. Long homologous arms may introduce unwanted mutations and increase the difficulty of plasmid construction. An arm length of greater than $0.3 \mathrm{~kb}$ gave a very high editing efficiency. Longer repair arms can increase the efficiency of gene editing but $300 \mathrm{bp}$ repair arms are long enough for genome deletion.

By assembling the $g f p$ gene into porB repair arms we also used this system to create a $g f p$ inserted mutation. The efficiency of gene insertion for $300 \mathrm{bp}$ repair arms was not as high as that for $1000 \mathrm{bp}$ arms (Additional file 1: Figure S2), possibly owning to the insert gene being longer than the repair arms. This low efficiency may be related to low homologous recombination efficiency. It is, therefore, better for the repair arms to be longer than the target gene for gene insertion.

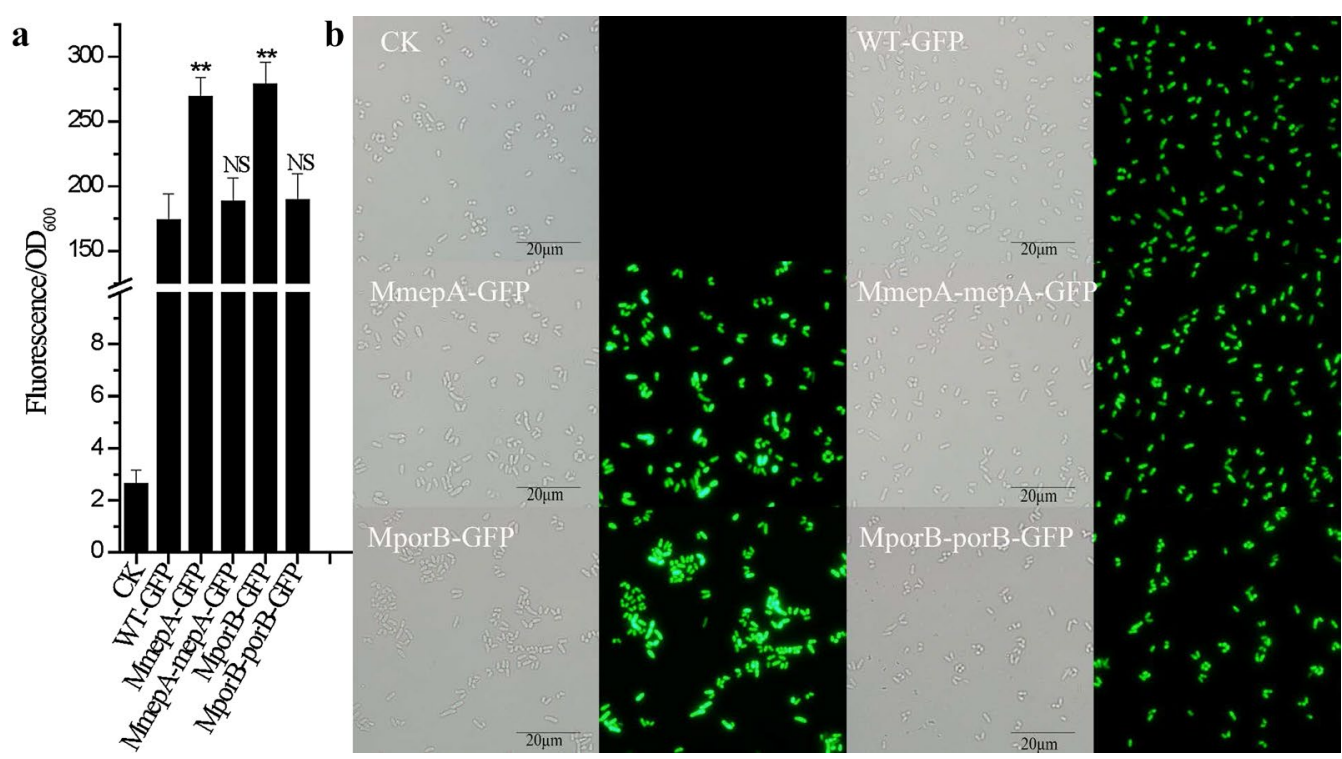

Fig. 7 GFP expression in the porB, mepA, clpX and Ncgl0911-deleted strains. CK is a negative control of the wild-type strain containing pXMJ19 without the gfp gene. WT-GFP is a positive control of the wild-type strain containing pXMJ19-EGFP; MmepA-GFP is the mepA-deleted mutant with plasmid PXMJ19-EGFP; MmepA-mepA-GFP is the mepA-deleted mutant complemented by mepA gene and containing pXMJ19-EGFP; MporB-GFP is the porB-deleted mutant with plasmid pXMJ19-EGFP; MporB-porB-GFP is the porB-deleted mutant complemented by porB gene and containing pXMJ19-EGFP. a Fluorescence intensities normalized against culture $\mathrm{OD}_{600}$ were used to indicate the expression of GFP. $\mathbf{b}$ The expression of GFP was determined by fluorescence microscopy with an exposure time of $1 \mathrm{~s}$. The mepA and porB mutants showed GFP expression enhanced by 55.2 and $62.4 \%$, respectively, relative to the wild type 
In addition, it is difficult to express some high molecular weight proteins in C. glutamicum; therefore, we codon-optimized the Cas 9 gene and an SD sequence was added in front of the ATG initiation codon. We found that no transformants were produced by the plasmid containing the de-repressed Ptac promoter. We speculate that Cas9 is toxic to C. glutamicum; therefore, we used the inducible Ptac promoter and a low concentration of IPTG to induce expression of Cas9. Jiang et al. [34] also found that Cas9 was toxic to C. glutamicum and they speculated that SpCas9 binds tightly to PAMs, even without a crRNA. In our study, the SNP and Indel were not detected in the C. glutamicum wild type strain containing the Cas9 without sgRNA compared with the wild type strain, indicating the toxic was not caused by the off target. We also found the transformants containing the Cas9 plasmid grew at a lower rate than the wild type. When the plasmid was cured, the growth rate of strains returned to the normal levels. Moreover, the Cas9 plasmid is easy to be lost without antibiotics. Therefore we can use this effect to cure the plasmid after performing gene editing.

In summary, the CRISPR/Cas9 system developed in this study will accelerate research into gene function, metabolic engineering, specific biosynthetic pathway analysis, and genetic modification for recombinant protein expression.

\section{Methods}

\section{Strains, plasmids, media and reagents}

All bacterial strains and plasmids used in this study are described in Additional file 1: Table S1. E. coli DH5 $\alpha$ was used as a cloning host for plasmid construction. The replicon initiator of pEC-XK99E was replaced with the temperature sensitive repA, which is from PDTW109 [9]. C. glutamicum ATCC 13032 was purchased from the American Type Culture Collection (ATCC). C. glutamicum CGMCC1.15647 was used as the host for foreign protein expression and was donated by Zhangjiagang Huachang Pharmaceutical Co. (Zhangjiagang City, China).

Escherichia coli, were cultured in LB medium (LuriaBertani: $10 \mathrm{~g} / \mathrm{L}$ tryptone, $5 \mathrm{~g} / \mathrm{L}$ yeast extract and $10 \mathrm{~g} / \mathrm{L}$ $\mathrm{NaCl})$ at $37^{\circ} \mathrm{C}$ with shaking at $200 \mathrm{rpm}$. C. glutamicum was cultured in LBB medium (LB supplemented with brain heart infusion: $10 \mathrm{~g} / \mathrm{L}$ tryptone, $5 \mathrm{~g} / \mathrm{L}$ yeast extract, $10 \mathrm{~g} / \mathrm{L}$ brain heart infusion Broth and $10 \mathrm{~g} / \mathrm{L} \mathrm{NaCl})$ at $30^{\circ} \mathrm{C}$ with shaking at $200 \mathrm{rpm}$. LBHIS (LB supplemented with brain heart infusion and sorbitol: $5 \mathrm{~g} / \mathrm{L}$ tryptone, $2.5 \mathrm{~g} / \mathrm{L}$ yeast extract, $18.5 \mathrm{~g} / \mathrm{L}$ brain heart infusion Broth, $91 \mathrm{~g} / \mathrm{L}$ sorbitol and $5 \mathrm{~g} / \mathrm{L} \mathrm{NaCl}$ ) medium was used for obtaining transformants of C. glutamicum. Antibiotics were added at the following concentrations: in E. coli, kanamycin $30 \mu \mathrm{g} / \mathrm{mL}$, ampicillin $100 \mu \mathrm{g} / \mathrm{mL}$, and chloramphenicol $30 \mu \mathrm{g} / \mathrm{mL}$; in C. glutamicum, kanamycin $10 \mu \mathrm{g} / \mathrm{mL}$, and chloramphenicol $10 \mu \mathrm{g} / \mathrm{mL}$.

Plasmid DNA was extracted using an AxyPrep Plasmid Miniprep kit (Axygen, Union City, CA, USA). DNA fragments from polymerase chain reactions (PCRs) and restriction enzyme digestions were purified using the AxyPrep Gel Extraction Kit (Axygen, Union City, CA, USA). Genomic DNA was extracted using a Bacterial genomic DNA Extraction Kit (TIANGEN, Beijing, China). Taq polymerase and T4 DNA ligase were purchased from Takara (Dalian, China). Restriction endonucleases were purchased from Thermo scientific (San Jose, CA, USA). Q5 and Gibson assembly kits were purchased from NEB (Beverly, MA, USA). Primers were purchased from Genweiz (Suzhou, China).

\section{sgRNA design}

The sgRNA candidate sequences target PAM recognition domains in the genome, and all candidate sgRNA-target sequences had N20NGG motifs. Target gene sequences were downloaded from NCBI GenBank and analyzed by Vector NTI software (Thermo Fisher Scientific) for selection of sgRNA PAM sites using GN19NGG as a motif. The $3^{\prime}$ ends of protospacers avoided the sequence TTTT, and the GC content was between 40 and $60 \%$. All protospacer candidates were blast searched against the NCBI C. glutamicum reference genome to identify sgRNA off-target sites that might produce off-target effects (https://blast.ncbi.nlm.nih.gov/Blast.cgi, Reference Sequence: NC_003450.3). All the sgRNA sequences (N20 sequences) and their PAM sites used are given in Table 1. Primers used are given in Additional file 1: Table S2.

\section{Plasmid construction}

The two-plasmid system, in which Cas9 and sgRNA are in separate plasmids, pFSC and pFST, was used for genome editing as shown in Fig. 1a and b. The Cas9 used in our system was codon-optimized for C. glutamicum using the Genweiz Sequence Analysis program to give a GC content of $52 \%$ compared with $35 \%$ in S. pyogens. The construction procedure of the pFSC plasmid was as follows: the codon-optimized cas 9 gene was PCR-amplified from plasmid pcas9. The SD sequence, which is indispensable for Cas9 expression, was contained in the primer. Then the fragment was subcloned into pXMJ19 using HindIII and EcoRI sites, to be under the control of the IPTG inducible Ptac promoter.

The temperature sensitive plasmid, pEC-XK99E, was selected as the backbone of pFST. The $20 \mathrm{nt}$ target sequence within the sgRNA scaffold was amplified from psgRNA (Fig. 1c). The sgRNA scaffold was flanked by EcoRI and $\mathrm{XbaI}$ restriction sites to allow easy insertion 
into pFST. sgRNA expression was inducible by IPTG under the control of the Ptrc promoter.

Left and right repair arms, from the $5^{\prime}$ and $3^{\prime}$ regions of the targeted genes, respectively, were amplified by PCR form C. glutamicum genomic DNA. The left arm was amplified by primers LF and LR, the right arm was amplified by primers RF and RR. The outer two primers (LF and RR) contained $20 \mathrm{bp}$ overhang regions of the $5^{\prime}$ and $3^{\prime}$ ends of the BglII site from the pFST plasmid, respectively, and the inner two primers (FR and RF) contained $10 \mathrm{bp}$ overhang regions of the other repair arm (Fig. 2a). After gel purification of the fragments and the $B g l I I-$ digested pFST plasmid, the left arm, right arm and plasmid were assembled using a Gibson assembly cloning kit (NEB). The construction of the pFST plasmid was verified by PCR using the primers pecBglF and pecBglR, and was confirmed by sequencing.

The construction of repair arms used to generate point mutations and gene insertions was the same as that of gene deletion. The mutation site was designed in the inner two primers. The insertion gene primers contained $10 \mathrm{bp}$ overhang regions of the left and right repair arms and arms were assembled using the Gibson assembly cloning kit (Fig. 4a, b).

\section{Genome editing}

Corynebacterium glutamicum competent cells were prepared using a previously reported method [9]. Electroporation was performed in a $1 \mathrm{~mm}$ gene pulser cuvette (Bio-Rad, USA) at $1.8 \mathrm{kV}$, Plasmids were added to $100 \mu \mathrm{L}$ competent cells that had been thawed on ice for $5 \mathrm{~min}$ and mixed gently to ensure even distribution. After electroporation, $1 \mathrm{~mL}$ LBHIS medium was immediately added to the cells he cell suspension and incubated for $6 \mathrm{~min}$ at $46{ }^{\circ} \mathrm{C}$ without shaking. The cells were incubated at $30{ }^{\circ} \mathrm{C}$ for $2 \mathrm{~h}$, spread onto LBHIS agar containing kanamycin $(10 \mu \mathrm{g} / \mathrm{mL})$, chloramphenicol $(10 \mu \mathrm{g} / \mathrm{mL})$ and IPTG $(0.01 \mathrm{mM})$, and then incubated $18 \mathrm{~h}$ at $30{ }^{\circ} \mathrm{C}$. Transformants were confirmed by PCR amplification of the cas 9 gene and the repair arm. To obtain the genedeletion strain directly, $1 \mu \mathrm{g}$ pFSC and pFST plasmids were coelectroporated, but the transformation efficiency was low. To increase the transformation efficiency, competent cells containing the pFSC plasmid can be obtained first, and then used for other round of transformation.

\section{Re-sequencing analysis}

Re-sequencing was performed to detect the off target in edited strains. Total DNA was extracted from C. glutamicum according to manufacturer's protocol (TIANGEN, Beijing, China). DNA quality was determined using Qubit Fluorometer (Thermo Fisher Scientific, San Jose, CA, USA) to determine total mass and Fragment Analyzer to determine DNA integrity. The genome of C. glutamicum was sequenced using an Illumina HiSeq 4000 system (Illumina, San Diego, CA, USA) at the Beijing Genomics Institute (Shenzhen, China). Genomic DNA was sheared randomly to construct three read libraries with lengths of (300 bp) by a Bioruptor ultrasonicator (Diagenode, Denville, NJ, USA) and physico-chemical methods. The paired-end fragment libraries were sequenced according to the Illumina HiSeq 4000 system's protocol. Raw reads of low quality from paired-end sequencing (those with consecutive bases covered by fewer than five reads) were discarded. The sequenced reads were assembled using SOAPdenovo v1.05 software.

\section{Plasmid curing}

To cure mutant strains of the pFST plasmid to enable their use in a second round of genome editing, the mutant strains were inoculated in $5 \mathrm{~mL}$ of LBB medium containing chloramphenicol $(10 \mu \mathrm{g} / \mathrm{mL})$. The culture was incubated at $30{ }^{\circ} \mathrm{C}$ overnight and the next day $50 \mu \mathrm{L}$ of culture was diluted 1:100 in $5 \mathrm{~mL} \mathrm{LBB}$, and incubated at $37{ }^{\circ} \mathrm{C}$ until the culture had visibly grown. Then cells were streaked onto an LBB plate and cultured overnight at $30{ }^{\circ} \mathrm{C}$. Colonies cured of knockout plasmid were confirmed by streaking them onto LBB plates containing kanamycin and by PCR analysis.

For curing edited clones of pFSC, colonies harboring pFSC were inoculated into $5 \mathrm{~mL}$ LBB medium and grown at $30{ }^{\circ} \mathrm{C}$ to an $\mathrm{OD}_{600}$ of 1.0 , and then $5 \mu \mathrm{L}$ IPTG $(100 \mathrm{mM} /$ $\mathrm{mL}$ ) was added. The culture was incubated overnight and streaked onto LBB plates without antibiotics and IPTG. The colonies were confirmed as cured by streaking them onto LBB plates containing chloramphenicol and by PCR analysis.

\section{Detection of GFP activity}

To evaluate recombinant protein expression in por $B$, $m e p A, \operatorname{clp} X$ and Ncgl0911 knock-out strains, we constructed a GFP expression plasmid, pXMJ19-EGFP, as follows. The GFP gene was amplified by PCR from a pEGFP-N1 template. The amplified fragment was ligated into HindIII and BamHI sites of pXMJ19. The lacIq gene was removed from pXMJ19 to make it a constitutive expression vector. The physical map of pXMJ19-EGFP is shown in Additional file 1: Figure S5. The resulting plasmid, pXMJ19-EGFP, was introduced into the four genedeleted C. glutamicum ATCC 13032 strains. To check the generated mutants by genetic complementation, plasmid pECXK99 was used as borne to expression the deleted gene. The lacIq gene was removed from pECXK99 to make it a constitutive expression vector. The deleted gene was amplified from the chromosome and was ligated into pECXK99. The physical map of pECXK99-gene is shown 
in Additional file 1: Figure S5. The GFP expression plasmid, pXMJ19-EGFP, was introduced into the complementation strains. These were then grown overnight in 24-well deep-well plates (LifeFeng, Hangzhou, China) containing $2 \mathrm{~mL}$ culture medium per well. Cultures were then diluted 1:100 into $2 \mathrm{~mL}$ fresh medium and grown for $24 \mathrm{~h}$. The $\mathrm{OD}_{600}$ and fluorescence intensity values were measured by a Synergy H4 microplate reader (BioTek, USA). The excitation of EGFP was at $488 \mathrm{~nm}$ and emission was at $507 \mathrm{~nm}$. Cultivation and measurements were performed in triplicate. Fluorescence intensities normalized against culture $\mathrm{OD}_{600}$ were used to indicate the expression level of GFP. Fluorescence intensity was also analyzed by fluorescence microscopy (OLYMPUS) with an exposure time of $1 \mathrm{~s}$. GFP protein was also detected by $12 \%(\mathrm{w} / \mathrm{v})$ sodium dodecyl sulfate-polyacrylamide gel electrophoresis (SDS-PAGE).

\section{Additional file}

Additional file 1. Additional Figures S1-S7 and Tables S1-S3.

\begin{abstract}
Abbreviations
crRNA: CRISPR RNA; tracrRNA: trans-activating CRISPR RNA; PAM: protospaceradjacent motif; DSB: double-strand break; NHEJ: non-homologous end joining; HDR: homolog-directed repair; sgRNA: synthetic guide RNA; GFP: green fluorescent protein; SNP: single nucleotide polymorphism; Indel: insertions and deletion.
\end{abstract}

\section{Authors' contributions}

$F P, X L$, and $Z B$ conceived the experiments. FP, XW, YS, and GD performed the experiment. $F P$ and $X W$ analyzed the results. $F P, X L$, and $Z B$ wrote and revised the paper. All authors read and approved the final manuscript.

\begin{abstract}
Author details
${ }^{1}$ National Engineering Laboratory for Cereal Fermentation Technology, Jiangnan University, Wuxi 214122, China. ${ }^{2}$ The Key Laboratory of Industrial Biotechnology, Ministry of Education, School of Biotechnology, Jiangnan University, Wuxi 214122, China. ${ }^{3}$ The Key Laboratory of Carbohydrate Chemistry and Biotechnology, Ministry of Education, School of Biotechnology, Jiangnan University, Wuxi 214122, China.
\end{abstract}

\section{Acknowledgements}

We thank prof. Xiaoyuan Wang for providing the pDTW109 vector.

\section{Competing interests}

The authors declare that they have no competing interests.

\section{Availability of data and materials}

We provide support if necessary data for publication of the article, supporting data is provided in Additional file 1. Re-sequencing data is available at SRA under the Accession Code SRP121314.

\section{Consent for publication}

All authors give consent to publish the research in Microbial Cell Factories.

\section{Ethics approval and consent to participate}

This manuscript does not contain any studies with human participants or animals performed by any of the authors.

\section{Funding}

This study was funded by the National Basic Research Program of China (973 Program) (Grant Number 2013CB733602),and the 111 Project (No. 111-2-06), a project Funded by the Priority Academic Program Development of Jiangsu Higher Education Institutions and the Natural Science Foundation of Jiangsu Province (Grant Number BK20150148). This paper was supported by Research Innovation Program for College Graduates of Jiangsu Province (KYLX16-0808) and Jiangsu Science and Technology under Grant BK20131181.

\section{Publisher's Note}

Springer Nature remains neutral with regard to jurisdictional claims in published maps and institutional affiliations.

Received: 22 May 2017 Accepted: 8 November 2017

Published online: 14 November 2017

\section{References}

1. Kallscheuer N, Vogt M, Stenzel A, Gätgens J, Bott M, Marienhagen J. Construction of a Corynebacterium glutamicum platform strain for the production of stilbenes and (2S)-flavanones. Metab Eng. 2016;38:47-55.

2. Becker J, Zelder O, Häfner S, Schröder H, Wittmann C. From zero to hero-design-based systems metabolic engineering of Corynebacterium glutamicum for L-lysine production. Metab Eng. 2011;13:159-68.

3. Shin KC, Sim DH, Seo MJ, Oh DK. Increased production of food-grade D-tagatose from D-galactose by permeabilized and immobilized cells of Corynebacterium glutamicum, a GRAS host, expressing D-galactose isomerase from Geobacillus thermodenitrificans. J Agric Food Chem. 2016;64:8146-53.

4. Srivastava P, Deb J. Gene expression systems in corynebacteria. Protein Expr Purif. 2005;40:221-9.

5. Yim SS, An SJ, Choi JW, Ryu AJ, Jeong KJ. High-level secretory production of recombinant single-chain variable fragment (scFv) in Corynebacterium glutamicum. Appl Microbiol Biotechnol. 2014;98:273-84.

6. Liu X, Yang Y, Zhang W, Sun Y, Peng F, Jeffrey L, Harvey L, McNeil B, Bai Z. Expression of recombinant protein using Corynebacterium glutamicum: progress, challenges and applications. Crit Rev Biotechnol. 2016;36:652-64.

7. Baumgart M, Unthan S, Rückert C, Sivalingam J, Grünberger A, Kalinowski J, Bott M, Noack S, Frunzke J. Construction of a prophage-free variant of Corynebacterium glutamicum ATCC 13032 for use as a platform strain for basic research and industrial biotechnology. Appl Environ Microbiol. 2013;79:6006-15

8. Schäfer A, Tauch A, Jäger W, Kalinowski J, Thierbach G, Pühler A. Small mobilizable multi-purpose cloning vectors derived from the Escherichia coli plasmids pK18 and pK19: selection of defined deletions in the chromosome of Corynebacterium glutamicum. Gene. 1994;145:69-73.

9. Hu J, Tan Y, Li Y, Hu X, Xu D, Wang X. Construction and application of an efficient multiple-gene-deletion system in Corynebacterium glutamicum. Plasmid. 2013;70:303-13.

10. Suzuki N, Tsuge Y, Inui M, Yukawa H. Cre/loxP-mediated deletion system for large genome rearrangements in Corynebacterium glutamicum. Appl Microbiol Biotechnol. 2005;67:225-33.

11. Choi JW, Yim SS, Kim MJ, Jeong KJ. Enhanced production of recombinant proteins with Corynebacterium glutamicum by deletion of insertion sequences (IS elements). Microb Cell Fact. 2015;14:207.

12. Cleto S, Jensen JV, Wendisch VF, Lu TK. Corynebacterium glutamicum metabolic engineering with CRISPR interference (CRISPRi). ACS Synth Biol. 2016;5:375-85.

13. Tong Y, Charusanti P, Zhang L, Weber T, Lee SY. CRISPR-Cas 9 based engineering of actinomycetal genomes. ACS Synth Biol. 2015;4:1020-9.

14. Bikard D, Jiang W, Samai P, Hochschild A, Zhang F, Marraffini LA. Programmable repression and activation of bacterial gene expression using an engineered CRISPR-Cas system. Nucleic Acids Res. 2013;41:7429-37.

15. Larson MH, Gilbert LA, Wang X, Lim WA, Weissman JS, Qi LS. CRISPR interference (CRISPRi) for sequence-specific control of gene expression. Nat Protoc. 2013;8:2180-96.

16. Doudna JA, Charpentier E. The new frontier of genome engineering with CRISPR-Cas9. Science. 2014;346:1258096.

17. Lemay ML, Tremblay DM, Moineau S. Genome engineering of virulent Lactococcal phages using CRISPR-Cas9. ACS Synth Biol. 2017;6:1351. 
18. Hsu PD, Lander ES, Zhang F. Development and applications of CRISPRCas9 for genome engineering. Cell. 2014;157:1262-78.

19. Huang H, Chai C, Li N, Rowe P, Minton NP, Yang S, Jiang W, Gu Y. CRISPR/ Cas9-based efficient genome editing in Clostridium ljungdahlii, an autotrophic gas-fermenting bacterium. ACS Synth Biol. 2016;5:1355-61.

20. Pohl C, Kiel JA, Driessen AJ, Bovenberg RA, Nygard Y. CRISPR/Cas9 based genome editing of Penicillium chrysogenum. ACS Synth Biol. 2016;5:754-64.

21. Shalem O, Sanjana NE, Hartenian E, Shi X, Scott DA, Mikkelsen TS, Heckl D, Ebert BL, Root DE, Doench JG. Genome-scale CRISPR-Cas9 knockout screening in human cells. Science. 2014;343:84-7.

22. Paquet D, Kwart D, Chen A, Sproul A, Jacob S, Teo S, Olsen KM, Gregg A, Noggle S, Tessier-Lavigne M. Efficient introduction of specific homozygous and heterozygous mutations using CRISPR/Cas9. Nature. 2016;533:125-9.

23. Richardson CD, Ray GJ, DeWitt MA, Curie GL, Corn JE. Enhancing homology-directed genome editing by catalytically active and inactive CRISPRCas9 using asymmetric donor DNA. Nat Biotechnol. 2016;34:339-44.

24. Zerbini F, Zanella I, Fraccascia D, Konig E, Irene C, Frattini LF, Tomasi M, Fantappie L, Ganfini L, Caproni E, et al. Large scale validation of an efficient CRISPR/Cas-based multi gene editing protocol in Escherichia coli. Microb Cell Fact. 2017;16:68

25. Chen W, Zhang Y, Yeo WS, Bae T, Ji Q. Rapid and efficient genome editing in Staphylococcus aureus by using an engineered CRISPR/Cas9 system. J Am Chem Soc. 2017;139:3790-5.

26. Jiang Y, Chen B, Duan C, Sun B, Yang J, Yang S. Multigene editing in the Escherichia coli genome via the CRISPR-Cas9 system. Appl Environ Microbiol. 2015;81:2506-14.

27. Xu T, Li Y, Shi Z, Hemme CL, Li Y, Zhu Y, Van Nostrand JD, He Z, Zhou J. Efficient genome editing in Clostridium cellulolyticum via CRISPR-Cas9 nickase. Appl Environ Microbiol. 2015;81:4423-31.

28. Zhao D, Yuan S, Xiong B, Sun H, Ye L, Li J, Zhang X, Bi C. Development of a fast and easy method for Escherichia coli genome editing with CRISPR Cas9. Microb Cell Fact. 2016;15:205.

29. DiCarlo JE, Norville JE, Mali P, Rios X, Aach J, Church GM. Genome engineering in Saccharomyces cerevisiae using CRISPR-Cas systems. Nucleic Acids Res. 2013;41:4336-43.

30. Zhang K, Duan X, Wu J. Multigene disruption in undomesticated Bacillus subtilis ATCC 6051a using the CRISPR/Cas9 system. Sci Rep. 2016;6:27943.

31. Belhaj K, Chaparro-Garcia A, Kamoun S, Patron NJ, Nekrasov V. Editing plant genomes with CRISPR/Cas9. Curr Opin Biotechnol. 2015;32:76-84.
32. Jiang W, Zhou H, Bi H, et al. Demonstration of CRISPR/Cas9/sgRNA-mediated targeted gene modification in Arabidopsis, tobacco, sorghum and rice. Nucleic Acids Res. 2013;41(20):e188. https://academic.oup.com/nar/ article/41/20/e188/2414851

33. Dong ZQ, Chen TT, Zhang J, Hu N, Cao MY, Dong FF, Jiang YM, Chen P, Lu C, Pan MH. Establishment of a highly efficient virus-inducible CRISPR/ Cas9 system in insect cells. Antivir Res. 2016;130:50-7.

34. Jiang Y, Qian F, Yang J, Liu Y, Dong F, Xu C, Sun B, Chen B, Xu X, Li Y. CRISPR-Cpf1 assisted genome editing of Corynebacterium glutamicum. Nat Commun. 2017;8:15179

35. Sun Y, Guo W, Wang F, Peng F, Yang Y, Dai X, Liu X, Bai Z. Transcriptome and multivariable data analysis of Corynebacterium glutamicum under different dissolved oxygen conditions in bioreactors. PLoS ONE. 2016;11:e0167156.

36. Jakoby M, Ngouoto-Nkili C-E, Burkovski A. Construction and application of new Corynebacterium glutamicum vectors. Biotechnol Tech. 1999;13:437-41.

37. Nešvera J, Pátek M. Tools for genetic manipulations in Corynebacterium glutamicum and their applications. Appl Microbiol Biotechnol. 2011;90:1641.

38. Marchand CH, Salmeron C, Bou Raad R, Meniche X, Chami M, Masi M, Blanot D, Daffe M, Tropis M, Huc E, et al. Biochemical disclosure of the mycolate outer membrane of Corynebacterium glutamicum. J Bacteriol. 2012;194:587-97.

39. Bott M, Brocker M. Two-component signal transduction in Corynebacterium glutamicum and other corynebacteria: on the way towards stimuli and targets. Appl Microbiol Biotechnol. 2012;94:1131-50.

40. Engels S, Schweitzer JE, Ludwig C, Bott M, Schaffer S. clpC and clpP1P2 gene expression in Corynebacterium glutamicum is controlled by a regulatory network involving the transcriptional regulators $\mathrm{ClgR}$ and $\mathrm{HspR}$ as well as the ECF sigma factor sigmaH. Mol Microbiol. 2004;52:285-302.

41. Moker N, Brocker M, Schaffer S, Kramer R, Morbach S, Bott M. Deletion of the genes encoding the MtrA-MtrB two-component system of Corynebacterium glutamicum has a strong influence on cell morphology, antibiotics susceptibility and expression of genes involved in osmoprotection. Mol Microbiol. 2004;54:420-38.

42. Trentini DB, Suskiewicz MJ, Heuck A, Kurzbauer R, Deszcz L, Mechtler K, Clausen T. Arginine phosphorylation marks proteins for degradation by a Clp protease. Nature. 2016;539:48-53.

\section{Submit your next manuscript to BioMed Central and we will help you at every step:}

- We accept pre-submission inquiries

- Our selector tool helps you to find the most relevant journal

- We provide round the clock customer support

- Convenient online submission

- Thorough peer review

- Inclusion in PubMed and all major indexing services

- Maximum visibility for your research

Submit your manuscript at www.biomedcentral.com/submit 